\title{
Luminous blue variables as the progenitors of supernovae with quasi-periodic radio modulations
}

\author{
R. Kotak ${ }^{1}$ and J. S. Vink ${ }^{2}$ \\ ${ }^{1}$ European Southern Observatory, Karl-Schwarzschild Str. 2, 85748 Garching bei München, Germany \\ e-mail: rkotak@eso.org \\ 2 Astrophysics group, Lennard-Jones Laboratories, Keele University, Staffordshire ST5 5BG, UK
}

Received 10 June 2006 / Accepted 1 October 2006

\section{ABSTRACT}

\begin{abstract}
The interaction between supernova ejecta and circumstellar matter, arising from previous episodes of mass loss, provides us with a means of constraining the progenitors of supernovae. Radio observations of a number of supernovae show quasi-periodic deviations from a strict power-law decline at late times. Although several possibilities have been put forward to explain these modulations, no single explanation has proven to be entirely satisfactory. Here we suggest that Luminous blue variables undergoing S-Doradus type variations give rise to enhanced phases of mass loss that are imprinted on the immediate environment of the exploding star as a series of density enhancements. The variations in mass loss arise from changes in the ionization balance of Fe, the dominant ion that drives the wind. With this idea, we find that both the recurrence timescale of the variability and the amplitude of the modulations are in line with the observations. Our scenario thus provides a natural, single-star explanation for the observed behaviour that is, in fact, expected on theoretical grounds.
\end{abstract}

Key words. stars: mass-loss - stars: winds, outflows - stars: supernovae: individual: SN 2001ig -

stars: supernovae: individual: SN 2003bg - stars: supernovae: individual: SN 1979C - stars: supernovae: individual: SN 1998bw

\section{Introduction}

Massive stars and core-collapse supernovae ( $\mathrm{SNe}$ ) play an important role in driving the chemical evolution of galaxies and in shaping the dynamics of the interstellar medium over all cosmological epochs, ever since the very first stars came into existence. Despite their importance, the lives and deaths of massive stars are poorly understood; in particular, it is not known with any degree of certainty which massive stars produce which SNe.

While progress is gradually being made in directly identifying core-collapse SN progenitors by searching for them in preexplosion images (e.g. Smartt 2002; van Dyk et al. 2003), such studies are limited either to extremely nearby $\mathrm{SNe}$ or to those occurring in uncrowded regions. Perhaps as a result of the stellar initial mass function, most direct detections of progenitors have yielded masses that are not significantly greater than $\sim 10 M_{\odot}$.

Although the evolution of the most massive stars (with $M>$ $30 M_{\odot}$ ) is largely unconstrained, it is generally accepted that mass loss drives these objects through the $\mathrm{O}$ star, luminous blue variable (LBV), and Wolf-Rayet (WR) phases (e.g. Chiosi \& Maeder 1986). Significant progress has been made in recent years in our understanding of the mass loss properties over the various evolutionary phases of massive stars. In a series of papers, Vink et al. (1999, 2000, 2001) have provided predictions of the mass-loss rates of $\mathrm{O}$ and B supergiants, LBVs (Vink \& de Koter 2002), and late-type WR-type stars as a function of metal content (Vink \& de Koter 2005).

Supernovae that result from the core-collapse of massive stars explode in environments that have been modified by mass loss from the progenitor. The SN ejecta interact first with this circumstellar material before interacting with interstellar material.
We might therefore expect that the distinct differences in wind properties over the lifespan of a massive star might be imprinted onto the resulting circumstellar media (CSM). Furthermore, we would then expect these differences to be manifested in the interaction between the SN ejecta and surrounding material. By quantifying these differences one can then constrain the evolutionary phase of the exploding object.

Over the past few decades, radio observations of $\mathrm{SNe}$ have provided a means of constraining the density of the CSM around core-collapse SNe. The inferred mass-loss rates from modelling most radio SN light curves yield values of $\dot{M} \sim$ $10^{-6}-10^{-4} M_{\odot} \mathrm{yr}^{-1}$ (e.g. compilation in Weiler et al. 2002). Unfortunately, these average mass-loss rates are generally only accurate to within a factor of $\sim 10$ and are typical of almost all types of massive star, making it difficult to pin down the evolutionary phase during which core-collapse occurred.

A small subset of radio $\mathrm{SNe}$, however, show quasi-periodic modulations in their radio lightcurves. In what follows, we argue that this type of modulation may be the result of an LBV star that underwent $S$ Dor variations that entailed opacity changes in the wind-driving region, resulting in varying mass-loss rates.

\section{LBV mass-loss variability}

Luminous blue variables are unstable massive stars, located in the upper portion $\left(\log \left(L / L_{\odot}\right) \gtrsim 5.5\right)$ of the Hertzsprung-Russell diagram (Humphreys \& Davidson 1994). They exhibit different types of variability that can be divided into three categories: (i) small-amplitude ( 0.1 mag) "micro" variability, which is common amongst blue supergiants; (ii) "moderate" S Doradus variations of $\sim 1-2 \mathrm{mag}$ (SD phases), and (iii) truly "giant" 
eruptions, of which P Cyg and Eta Car are the best-known examples. According to an extensive list compiled by van Genderen (2001), the SD-phases occur on two timescales: <10 yrs ("short SD" phases) and >20 yrs ("long SD" phases).

Spectroscopically-determined LBV mass-loss rates are found to change on S Dor time-scales (e.g. Stahl et al. 2001, 2003). Vink \& de Koter (2002) attribute this mass-loss variability to the ionization and recombination of the Fe lines that are responsible for driving the wind. This leads to "bi-stable" behaviour in the wind (Pauldrach \& Puls 1990; Lamers et al. 1995; Vink et al. 1999) causing the star to flip back and forth between two states: from low mass-loss, high-velocity winds to high mass-loss, low velocity winds. This bi-stable behaviour results in jumps in the mass-loss rate of a factor of $\sim 2$ for LBVs (Vink \& de Koter 2002) and a decrease by a factor of $\sim 2$ in the terminal wind velocity between $\mathrm{O}$ and $\mathrm{B}$ supergiants (Lamers et al. 1995). The wind density would therefore be expected to change by a factor of $\sim 4$ on the timescale of the $S$ Doradus variations. In the absence of any other material around the star, this would result in a pattern of concentric shells of varying density.

\section{Radio SN lightcurves and stellar wind interaction}

The properties of Radio $\mathrm{SNe}$ ( $\mathrm{RSNe}$ ) lightcurves and the model for the interaction of the SN blastwave with the surrounding CSM as described by Chevalier (1982a,b) has been reviewed by Weiler et al. (2002). In short, the source switches on, reaches a peak, and generally decays smoothly on a timescale of years. The peak is attained at different times (days to months) for different radio frequencies, depending on the optical depth of either external and/or internal absorbing material. For a discussion of the relative importance of free-free absorption (FFA) by an external - possibly clumpy - medium, versus synchrotron selfabsorption (SSA), we refer the reader to Chevalier (1998) and Fransson \& Björnsson (1998). The radio emission nevertheless places constraints on the density $\rho$, and therefore on the ratio of $\dot{M}$ to the terminal wind velocity $\left(v_{\mathrm{w}}\right)$ of the CSM of the SN progenitor: $\rho \propto \dot{M} / v_{\mathrm{w}} r^{2}$. Using RSNe lightcurves, and generally assuming a typical $v_{\mathrm{w}}$ for a red (super)giant $\left(\sim 10-20 \mathrm{~km} \mathrm{~s}^{-1}\right)$, constraints on $\dot{M}$ of pre-SN winds have been placed on about a dozen RSNe (see Table 3, Weiler et al. 2002) with typical $\dot{M}$ values in the range $10^{-6}-10^{-4} M_{\odot} \mathrm{yr}^{-1}$.

The shock radius can be constrained through radio observations, whilst the measured variability timescale is set by the wind velocity of the progenitor (see below). Ideally, one would wish to spatially resolve the shock radius directly, as was possible for SN 1979C using VLBI techniques (Bartel \& Bietenholz 2003). Usually, one needs to assess the relative importance of SSA vs. FFA. If SSA prevails, and if the peak flux is available, i.e., at the transition between the optically thick to thin phases, assuming equipartition between the magnetic fields and relativistic electrons yields an estimate of the size of the radio-emitting region (Shklovskii 1985; Chevalier 1998). If FFA is dominant, it is usual to simply adopt the measured velocity from optical lines at early times, together with parameters from modelling the light curves at different frequencies (Weiler et al. 2002).

\section{Modulations in radio $\mathrm{SNe}$ lightcurves}

Variations in the radio lightcurves have been reported for about a dozen $\mathrm{SNe}$ of both type II and type Ibc (see Table 4 of Soderberg et al. 2005). These can generally be interpreted as due to variations in the mass-loss rate due to a changing evolutionary phase of the SN progenitor. A subset of radio SNe show episodic bumps. These include the classic cases of SN 1979C (Weiler et al. 1992) and, of SN 1998bw (Kulkarni et al. 1998) which was associated with GRB 980425 (Galama et al. 1998), as well as two recent radio SNe, SN 2001ig (Ryder et al. 2004) and SN 2003bg (Soderberg et al. 2005). This has brought the number of radio $\mathrm{SNe}$ that show recurrent modulations in their radio light curves, to four. A caveat to the above is that finer sampling of radio light curves may reveal the extent to which such behaviour is commonplace. Interestingly, SNe 2001ig and 2003 bg are strikingly similar in terms of both the amplitudes and timescales of the variations (see Fig. 8 in Soderberg et al. 2005, for a comparison). We focus our discussion on these two $\mathrm{SNe}$ and defer discussion of SN 1979C and 1998bw to Sect. 4.3.

\subsection{SN $2001 \mathrm{ig}$}

SN 2001ig is a transition object. On the basis of optical spectroscopy, it was initially classified as type II (i.e. showing $\mathrm{H}$ lines) by Matheson \& Jha (2001) but metamorphosed into a type Ib/c object (i.e. no H lines, weak He lines) by Filippenko (2002) about 9 months later. This suggests that it has lost most of its H-rich envelope (Ryder et al. 2004). We will return to this point in Sect. 5.

For SN 2001ig, Ryder et al. (2004) used the model of Weiler et al. (2002) to derive parameter fits to the radio lightcurves yielding parameters such as the radio spectral index $\alpha$, the decline rate of the optically thin phase $\beta$, and importantly, at least one parameter $\delta(=-2.56)$ that describes how the optical depth of the local CSM changes with time. The relevance of $\delta$ is that it relates to the rate at which the $\mathrm{SN}$ blast-wave radius $r_{\mathrm{BW}}$ changes with time: $r_{\mathrm{BW}} \propto t^{m}$ with $m=-\delta / 3$. In the mini-shell model by Chevalier (1982a,b), $m=1$ implies a non-decelerating CSM. For SN 2001ig, $m=0.85$.

The recurrence timescale $(t)$ of the bumps in the radio flux of SN 2001 ig is derived to be 150 days. Using Eq. (13) from Weiler et al. (1986),

$\Delta P=\frac{R_{\text {shell }}}{v_{\mathrm{w}}}=\frac{v_{\text {ejecta }} t_{\mathrm{i}}}{v_{\mathrm{w}} m}\left(\frac{t}{t_{\mathrm{i}}}\right)^{m}$

where $t_{\mathrm{i}}$ is set by when the ejecta velocity was measured. For SN 2001ig, $t_{\mathrm{i}} \sim 14 \mathrm{~d}$ and $v_{\text {ejecta }} \sim 15000 \mathrm{~km} \mathrm{~s}^{-1}$ (Clocchiatti $\&$ Prieto 2001). We stress that the dependence on $t_{i}$ is weak. Assuming $v_{\mathrm{w}}=10-20 \mathrm{~km} \mathrm{~s}^{-1}$, typical wind velocities for red (super)giants, Ryder et al. (2004) found a period $P$ between successive mass-loss episodes that was too long for red (super)giant pulsations (typically a few 100 days) and too short for thermal pulses $\left(10^{2}-10^{3}\right.$ years $)$. For these reasons, they invoked an edgeon, eccentric binary scenario, consisting of a WR-star and a massive companion. The configuration of the binary system would lead to a pile-up of mass, giving rise to modulations in the radio light curve. Such a scenario was proposed on the basis of hydrodynamical simulations by Schwarz \& Pringle (1996) to explain the radio lightcurve of SN 1979C.

One of the main differences between LBV and red giant winds is that LBV winds are faster, typically $100-500 \mathrm{~km} \mathrm{~s}^{-1}$ (e.g. Leitherer 1997) cf. 10-20 $\mathrm{km} \mathrm{s}^{-1}$ for red (super)giants. This implies that if the progenitor of SN 2001ig were an LBV, the expected period between successive mass-loss episodes would be $\Delta P \sim 25 \mathrm{yr}$ (for an assumed $v_{\mathrm{w}}=200 \mathrm{~km} \mathrm{~s}^{-1}$ ), consistent with the long $\mathrm{SD}$ phase. Inconsistencies in the derivation of $\Delta P$ in Ryder et al. (2004) results in their inferred radial spacings between the shells being too small $\left(\sim 2 \times 10^{15} \mathrm{~cm}\right.$, with $t_{i}=1 \mathrm{~d} \mathrm{cf}$. $1.6 \times 10^{16} \mathrm{~cm}$ from above) and might therefore be incompatible with the kind of WR pinwheel nebula they describe. 


\subsection{SN 2003bg}

SN 2003bg was classified as a type Ic (no H, He, Si lines) by Filippenko (2003); in less than a month, the spectrum of SN 2003bg had evolved to a type II SN, showing broad P Cygni lines of hydrogen (Hamuy et al. 2003). Soderberg et al. (2005) are able to fit the radio light curve under the assumption of SSA.

Remarkably, the short-term variability in the multifrequency radio lightcurves of $\mathrm{SN} 2003 \mathrm{bg}$ is found to be on very similar timescales as SN 2001ig. Soderberg et al. (2005) infer enhancements of a factor of $\sim 2$ in density during the deviations from a pure power-law evolution. They consider a range of possibilities that might account for the observed modulations in the lightcurve, but favour a single-star progenitor model of a WR star that underwent episodes of intensified mass loss. However, they do not specify the nature of the physical mechanism that might give rise to such periods of enhanced mass loss. Using the measured expansion velocity for SN 2003bg at a time $t_{\mathrm{i}} \sim 20$ days (Filippenko 2003; Hamuy et al. 2003), we find $\Delta P \sim 25$ years, again, consistent with the long SD phase.

\subsection{The cases of SN 1979C and SN 1998bw}

As outlined above, the relevance of SNe 2001ig and 2003bg-like events is that there is evidence of regularly changing mass loss for the SN progenitor. These observations may finally open up the possibility of yielding more stringent constraints on the preSN mass-loss properties than available from previous radio data. To our knowledge, the only massive stars known to fulfil the criterion of quasi-regularly spaced mass-loss episodes are LBVs.

The only other SNe that have shown episodic modulations in their radio lightcurves are SN 1979C and SN 1998bw. For SN 1979C, Weiler et al. (1992) found a radio modulation on a timescale of $\sim 1575$ days, i.e. a factor $\sim 10$ longer than for SNe 2001ig/2003bg. However, when we account for the rate at which the blast-wave radius changed with time, $\delta=$ -2.94 (Montes et al. 2000), we find $\Delta P \sim 160 \mathrm{yrs}$ for $v_{\text {ejecta }}$ $\sim 8000 \mathrm{~km} \mathrm{~s}^{-1}$ (Panagia et al. 1980). Now the circumburst radius of SN 1979C has been resolved with VLBI. Using the value thus derived of the radio size (Fig. 5, Bartel \& Bietenholz 2003), combined with the epoch of the radio modulations at $\sim 4$ and 8 years, we find a difference in angular size of $\sim 0.5$ mas. Assuming a distance of 15.2 Mpc (Freedman et al. 2001) then implies massloss variability on timescales of $\sim 150$ years $\left(v_{\mathrm{w}}=200 \mathrm{~km}\right.$ $\mathrm{s}^{-1}$ ), i.e. consistent with $\Delta P$ derived above, but longer than for $\mathrm{SNe} 2001 \mathrm{ig} / 2003 \mathrm{bg}$. We note as an aside that van Genderen (2001) does list some LBVs with SD phases that are greater than $\sim 100$ years.

Interestingly, for the more exotic case of SN 1998bw, and with a similar analysis to that in Sect. 4.1, with $m=0.78$ (Weiler et al. 2001) and a timescale of $t \simeq 50$ days, we find $\Delta P \sim 6$ years, which is consistent with the short SD timescale. We note, however, that the episodic bumps in the lightcurve of SN 1998bw are not as well-defined as the sinusoidal variations of SNe 2001ig/2003bg.

\section{Discussion}

We have proposed that LBVs undergoing S Doradus-type variations are viable progenitors of those $\mathrm{SNe}$ that show quasisinusoidal variations in their radio lightcurves. The main motivation for this suggestion is that $\mathrm{S}$ Doradus-type winds provide a natural and physical mechanism that could give rise to the observed radio modulations. Both the timescales and the amplitude of the variations in CSM density as a result of the S Doradus variations are in line with the constraints imposed by the radio SN data. Our proposed scenario therefore provides support for a single-star progenitor system. Admittedly, there are uncertainties associated with current analyses of the timescales of the radio $\mathrm{SNe}$ described in this paper, as well as with LBV S Dor variations in general. Until a significant body of radio $\mathrm{SNe}$ with episodic modulations becomes available, statistical considerations are deemed premature. If shorter SD variations are superimposed on the longer ones, then these might be revealed by further increasing the cadence of radio observations to a nightly basis.

It may be relevant that both $\mathrm{SNe} 2001$ ig and 2003bg are transitional objects. SN 2001ig is an example of the group of IIb $\mathrm{SNe}$, which switch from type II ( $\mathrm{H}$ and He both present) to type Ib (He present, H absent), whilst 2003bg was first classified as a type Ic, but then underwent a change to a type II. These facts suggest that SN2001ig and 2003bg have a rather limited amount of $\mathrm{H}$. This is also consistent with an LBV scenario, as LBV atmospheres are H-rich compared to WR stars, but He-rich compared to OB stars and red supergiants.

We do not exclude the possibility that objects like $\mathrm{SNe}$ 2003bg and 1998bw are in fact "stripped core" WR stars by the time they explode. However, given that WR stars are not known to exhibit the type of variability that we consider here, we believe it is more likely that the observed quasi-sinusoidal variations in their radio lightcurves are attributable to their prior LBV phase. This would mean that these objects are WR stars for only a very brief amount of time before they explode, and to all intents and purposes, we can categorise these objects as LBVs.

The fact that our proposed scenario is simple and workable does not mean other scenarios need necessarily be excluded. Ryder et al. (2006) recently found evidence for a binary companion of SN 2001ig. This in itself is not surprising, since it is well-known that a large fraction of massive stars occur in binary systems (e.g. Mason et al. 1998). The more relevant question of whether the potential binary companion ever had any direct influence on the star that exploded as SN 2001ig is more difficult to answer.

The binary scenario that could give rise to a pinwheel nebula as proposed by Ryder et al. $(2004,2006)$ and Schwarz \& Pringle (1996) requires the binary to be eccentric and viewed close to edge-on. Given the striking similarity of SN 2003bg to SN 2001ig, Soderberg et al. (2005) argue that the Wolf-Rayet pinwheel nebula hypothesis is unlikely, as the configurations of both systems must be very similar. They therefore propose a single WR progenitor, but do not put forward a mechanism for the CSM variability. The LBV progenitor scenario presented here may remove this shortcoming.

Acknowledgements. We thank the anonymous referee for constructive criticism, and Dr. S. Ryder for an interesting seminar on SN 2001ig, which stimulated this work. J.S.V. acknowledges financial support from an RCUK fellowship. R.K. acknowledges support from an ESO fellowship.

\section{References}

Bartel, N., \& Bietenholz, M. F. 2003, ApJ, 591, 301

Chevalier, R. A. 1982a, ApJ, 258, 790

Chevalier, R. A. 1982b, ApJ, 259, L85

Chevalier, R. A. 1998, ApJ, 499, 810

Chiosi, C., \& Maeder, A. 1986, ARA\&A, 24, 329

Clocchiatti, A., \& Prieto, J. L., 2001, IAU Circ. 7781

Filippenko, A. V., \& Chornock, R. 2002, IAU Circ. 7988

Filippenko, A. V., \& Chornock, R. 2003, IAU Circ. 8084

Fransson, C., \& Björnsson, C-I. 1998, ApJ, 509, 861 
Freedman, W. L., Madore, B. F.; Gibson, B. K. et al. 2001, ApJ, 553, 47

Galama, T. J., Vreeswijk, P. M., van Paradijs, J., et al. 1998, Nature, 395, 670

Hamuy, M., Philips, M., \& Thomas-Osip, J. 2003, IAU Circ. 8088

Humphreys, R. M., \& Davidson, K. 1994, PASP, 106, 1025

Kulkarni, S. R., Frail, D. A., Wieringa, M. H., et al. 1998, Nature, 395, 663

Lamers, H. J. G. L. M., Snow, T. P., \& Lindholm, D. M., 1995, ApJ, 455, L269

Leitherer, C. 1997, in LBVs: Massive Stars in Transition, eds. Nota \& Lamers, ASP Conf., 120, 58

Mason, B. D., Gies, D. R., Hartkopf, W. I., et al. 1998, AJ, 115, 821

Matheson, T., \& Jha, S. 2001, IAU Circ., 7772

Montes, M. J., Weiler, K. W., Van Dyk, S. D., et al. 2000, ApJ 532, 1124

Panagia, N., Vettolani, G., Boksenberg, A., et al. 1980, MNRAS 192, 861

Patat, F., Cappellaro, E., \& Danziger, J. 2001, ApJ 555, 900

Pauldrach, A. W. A., \& Puls, J. 1990, A\&A, 237, 409

Ryder, S. D., Sadler, E. M., Subrahmanyan, R., et al. 2004, MNRAS, 349, 1093

Ryder, S. D., Murrowood, C. E., \& Stathakis, R. A.,

[arXiv: astro-ph/0603336]

Schwarz, D. H., \& Pringle, J. E. 1996, MNRAS, 282, 1018
Shklovskii, I. S. 1985, Soviet Astr. Lett., 11, 105

Smartt, S. J. 2002, Ap\&SS, 565, 1089

Soderberg, A. M., Chevalier, R. A., Kulkarni, S. R., \& Frail, D. A. 2005, [arXiv: astro-ph/0512413]

Stahl, O., Jankovics, I., Kovács, et al. 2001, A\&A, 375, 54

Stahl, O., Gäng, T., Sterken, C., et al. 2003, A\&A, 400, 279

Tully, R. B. 1988, Nearby Galaxies Catalogue (Cambridge Univ. Press)

van Dyk, S., Li, W., Filippenko, A. V. 2003, PASP, 115, 1

van Genderen, A. M. 2001, A\&A, 366, 508

Vink, J. S., de Koter, A., \& Lamers, H. J. G. L. M. 1999, A\&A, 350, 181

Vink, J. S., de Koter, A.,\& Lamers, H. J. G. L. M. 2000, A\&A, 362, 295

Vink, J. S., de Koter, A., \& Lamers, H. J. G. L. M. 2001, A\&A, 369, 574

Vink, J. S., \& de Koter, A. 2002, A\&A,393, 543

Vink, J. S., \& de Koter, A. 2005, A\&A, 442, 587

Weiler, K., Sramek, R. A., Panagia, N., et al. 1986, ApJ, 301, 790

Weiler, K., Van Dyk, S. D., Pringle, J. E., \& Panagia, N. 1992, ApJ, 399, 672

Weiler, K., Panagia, N., \& Montes, M. J. 2001, ApJ, 562, 670

Weiler, K., Panagia, N., Montes, M., \& Sramek, R. A. 2002, ARA\&A, 40, 387 